\title{
Signals of neutralino dark matter from Earth and Sun
}

\author{
A. Bottino ${ }^{a}$, N. Fornengo ${ }^{a}$, G. Mignola ${ }^{a}$, L. Moscoso ${ }^{b}$ \\ a Dipartimento di Fisica Teorica, Università di Torino and INFN, Sezione di Torino, via P.Giuria I, 10125 Torino, Italy ${ }^{1}$ \\ b DAPNIA/DSM/SPP, CE-Saclay, 91191 Gif-sur-Yvette, France ${ }^{2}$
}

Received 13 July 1994

\begin{abstract}
We evaluate the fluxes of up-going muons detectable in a neutrino telescope and due to the annihilation of relic neutralinos in the Earth and in the Sun, taking realistically into account the fact that neutralinos might provide only a fraction of the local (solar neighbourhood) dark matter (DM). We determine the relation between the exposure At of the neutrino telescope ( $A$ being the telescope area, $t$ the life time) and the explorable range of the neutralino mass and composition, when the signal-to-background ratio is optimized by an appropriate angular selection.
\end{abstract}

\section{Introduction}

The perspectives of observing, with neutrino telescopes of large area, indirect signals of relic neutralinos form celestial bodies (Earth and Sun) are very appealing and are currently the subject of much investigation [1-3]. The sequence of the physical processes which could produce these signals are: i) capture of relic neutralinos by the macroscopic bodies; ii) subsequent accumulation of these particles in a region around the centre of these celestial objects; iii) pair annihilation of the accumulated neutralinos which would generate, by decay of the particles produced in the various annihilation final states, an output of high-energy neutrinos. These neutrinos may be detected in a neutrino telescope in two ways: either by looking at events due to the $\nu$-interactions inside the detector (contained events) or by measuring the muons which are produced by $\nu_{\mu}$ and $\bar{\nu}_{\mu}$ interactions in the rock around the detector and then traverse it up-

\footnotetext{
${ }^{1}$ E-mail addresses: (bottino, fornengo, mignola) @to.infn.it

${ }^{2}$ E-mail addresse: moscoso@hep.saclay.cea.fr
}

wardly. In the present paper we only discuss the latter case; we address the problem of the theoretical predictions for the fluxes of these up-going muons in a neutrino telescope and of their discrimination against the background due to atmospheric neutrinos. The present analysis aims at improving the previous ones in many instances: a) The calculations of the predicted signals for both macroscopic bodies (Earth and Sun) are presented in a common theoretical framework; in particular, this enables a comparative discussion about the informations obtainable by measurements of the two fluxes. b) It is realistically taken into account the fact that neutralinos might provide only a fraction of the local (solar neighbourhood) dark matter (DM) density. In order to establish whether and by how much the total DM density has to be rescaled, the neutralino relic abundance is explicitly evaluated at any point of the model parameter space where signals are calculated.c) It is finally discussed the relation between the exposure $A t$ of a neutrino telescope ( $A$ being the telescope area, $t$ the live time) and the explorable range of the neutralino mass (and composition), taking into 
account the optimization of the signal-to-background ratio $(\mathrm{S} / \mathrm{B})$.

The theoretical framework adopted in the present paper is the one provided by the Minimal Supersymmetric extension of the Standard Model (MSSM) [4], which, implemented by a general grand unification relation, may be formulated (for the neutralino sector) in terms of only three parameters: two mass parameters $M_{2}$ and $\mu$, and a third parameter $\tan \beta$ defined as $\tan \beta=v_{u} / v_{d}$ ( $v_{u}$ and $v_{d}$ are the v.e.v.'s that give masses to the up-type and down-type quarks, respectively). The neutralino, $\chi$, is defined to be the lowestmass linear combination of the two gauginos (photino and zino) and the two higgsinos

$$
\chi=a_{1} \tilde{\gamma}+a_{2} \tilde{Z}+a_{3} \tilde{H}_{1}^{0}+a_{4} \tilde{H}_{2}^{0} .
$$

Here $\tilde{\gamma}$ and $\tilde{Z}$ are the fields obtained from the original fields for the $U(1)$ and SU(2) neutral gauginos, $\tilde{B}$ and $\tilde{W}_{3}$, by a rotation in terms of the Weinberg angle.

It is useful to introduce a composition parameter $P=a_{1}^{2}+a_{2}^{2}$ which gives the gaugino fractional weight. The neutralino mass $m_{\chi}$ and the coefficients $a_{i}$ 's depend on the three model parameters $M_{2}, \mu$ and $\tan \beta$. Since almost everywhere in the parameter space there is a one-to-one correspondence: $\left(M_{2}, \mu\right) \leftrightarrow\left(P, m_{\chi}\right)$, at fixed $\tan \beta$, in the following we will use as a set of independent parameters: $P, m_{X}$ and $\tan \beta$. This allows us to present our results in terms of two quantities, $P$ and $m_{\chi}$, which have a direct physical meaning for the neutralino. In the following, for $\tan \beta$, unless differently specified, we will use the representative value $\tan \beta=8$.

We still have to state which values we assign to the unknown masses of the Higgs bosons and of the sfermions; in fact these masses enter in the theoretical calculations, since Higgses and sfermions play a crucial role in the physical processes initiated by neutralinos. As for the neutral Higgs bosons, we recall that in the MSSM there are three neutral Higgs particles: two CP-even bosons $h$ and $H$ (of masses $m_{h}, m_{H}$ with $m_{H}>m_{h}$ ) and a CP-odd one $A$ (of mass $m_{A}$ ). Once a value for one of these masses (say, $m_{h}$ ) is assigned, the other two masses $\left(m_{A}, m_{H}\right)$ are derived through mass relationships depending on radiative effects. In the present paper the Higgs mass $m_{h}$ is set at the value of $50 \mathrm{GeV}$. As for the sfermion masses, these are set here at the value $m_{\tilde{f}}=1.2 m_{\chi}$, when $m_{X}>45$
$\mathrm{GeV}, m_{\tilde{f}}=45 \mathrm{GeV}$ otherwise. Only the mass of the stop quark is assigned a larger value of $1 \mathrm{TeV}$. The top mass is fixed at $m_{t}=170 \mathrm{GeV}$.

\section{Neutralino local density}

An important quantity that enters in the capture rate of relic neutralinos by macroscopic bodies is the neutralino local density $\rho_{X}$. One can assume that $\rho_{X}$ is equal to the local value of the total DM density $\rho_{l}$, when the neutralino relic abundance $\left(\Omega_{\chi} h^{2}\right)$ turns out to be at the level of an $\left(\Omega h^{2}\right)_{\min }$ consistent with $\rho_{l}$. On the contrary when $\left(\Omega_{\chi} h^{2}\right)$ is smaller than $\left(\Omega h^{2}\right)_{\text {min }}$ the value to be assigned to $\rho_{\chi}$ has to be appropriately reduced. Thus we evaluate $\Omega_{X} h^{2}$ and we determine $\rho_{X}$ by adopting a standard rescaling procedure [5]:

$$
\begin{aligned}
& \rho_{X}=\rho_{l}, \quad \text { when } \Omega_{X} h^{2} \geq\left(\Omega h^{2}\right)_{\min }, \\
& \rho_{X}=\rho_{l} \frac{\Omega_{X} h^{2}}{\left(\Omega h^{2}\right)_{\min }}, \quad \text { when } \Omega_{\chi} h^{2}<\left(\Omega h^{2}\right)_{\min } .
\end{aligned}
$$

Here $\left(\Omega h^{2}\right)_{\min }$ is set equal to 0.03 . For $\rho_{l}$, we have used the value $\rho_{l}=0.3 \mathrm{GeV} \mathrm{cm}^{-3}$.

The neutralino relic abundance $\Omega_{\chi} h^{2}$ is evaluated here following the procedure illustrated in Ref. [6]. By way of example in Fig. 1 we display $\Omega_{\chi} h^{2}$ as a function of $m_{\chi}$ for three representative neutralino compositions: i) a gaugino-dominated composition ( $P=$ 0.9 ), ii) a composition of maximal gaugino-higgsino mixing $(P=0.5)$, iii) a higgsino-dominated composition ( $P=0.1)$. As expected, out of the three compositions displayed in Fig. 1, the gaugino-dominated state provides the largest values of $\Omega_{\chi} h^{2}$. More substantial values of $\Omega_{\chi} h^{2}$ occurs if one considers purer gaugino compositions $(P \gtrsim 0.99)$, as shown in Fig. 2 , where, together with the reference value $P=0.5$, are also displayed the rather extreme cases: $P=0.01$ (very pure higgsino composition) and $P=0.99$ (very pure gaugino composition). The very pronounced dips in the plots of Figs. 1,2 are due to the s-poles in the $\chi-\chi$ annihilation cross section (exchange of the $Z$ and of the neutral Higgs bosons).

Displayed in Fig. 3 are the values of $\Omega_{\chi} h^{2}$ versus $m_{\chi}$ in the form of scatter plots obtained by varying $M_{2}$ and $\mu$ over a grid of constant spacing in a log-log plane over the ranges $20 \mathrm{GeV} \leq M_{2} \leq 6 \mathrm{TeV}, 20 \mathrm{GeV}$ 


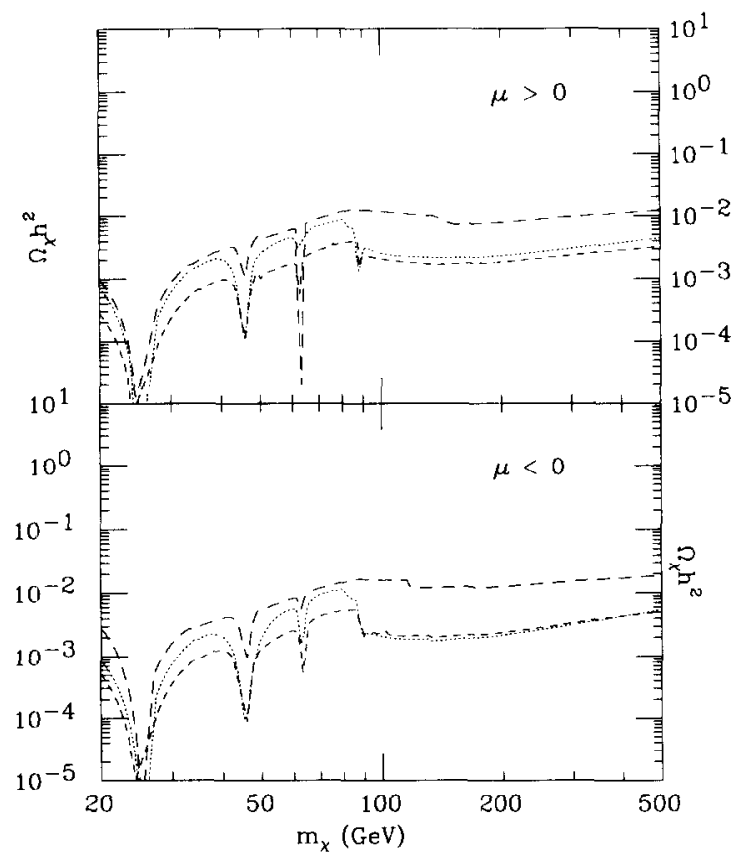

Fig. 1. Neutralino relic abundance $\Omega_{\chi} h^{2}$ as a function of $m_{\chi}$ for three different neutralino compositions $P=0.1$ (dotted line), 0.5 (short-dashed line), 0.9 (long-dashed line). The upper plot refers to positive values of $\mu$, the lower to negative values.

$\leq|\mu| \leq 3 \mathrm{TeV}$. By comparing Fig. 3 with Fig. 2 one sees that the large spread in values for $\Omega_{\chi} h^{2}$, displayed in Fig. 3 , is due to configurations of extremely pure composition.

\section{Capture rates and annihilation rates}

The capture rate $C$ of the relic neutralinos by a macroscopic body may be evaluated by the standard formula [7]

$$
C=\frac{\rho_{X}}{v_{X}} \sum_{i} \frac{\sigma_{\mathrm{el}, i}}{m_{X} m_{i}}\left(M_{B} f_{i}\right)\left\langle v_{e s c}^{2}\right\rangle_{i} X_{i},
$$

where $v_{X}$ is the neutralino mean velocity, $\sigma_{\text {el. } i}$ is the cross section of the neutralino elastic scattering off the nucleus $i$ of mass $m_{i}, M_{B} f_{i}$ is the total mass of the element $i$ in the body of mass $M_{B},\left\langle v_{e s c}^{2}\right\rangle_{i}$ is the square escape velocity averaged over the distribution of the element $i, X_{i}$ is a factor which takes account of kinematical properties occurring in the neutralino-nucleus interactions. The elastic $\mathcal{X}$-nucleus cross sections are

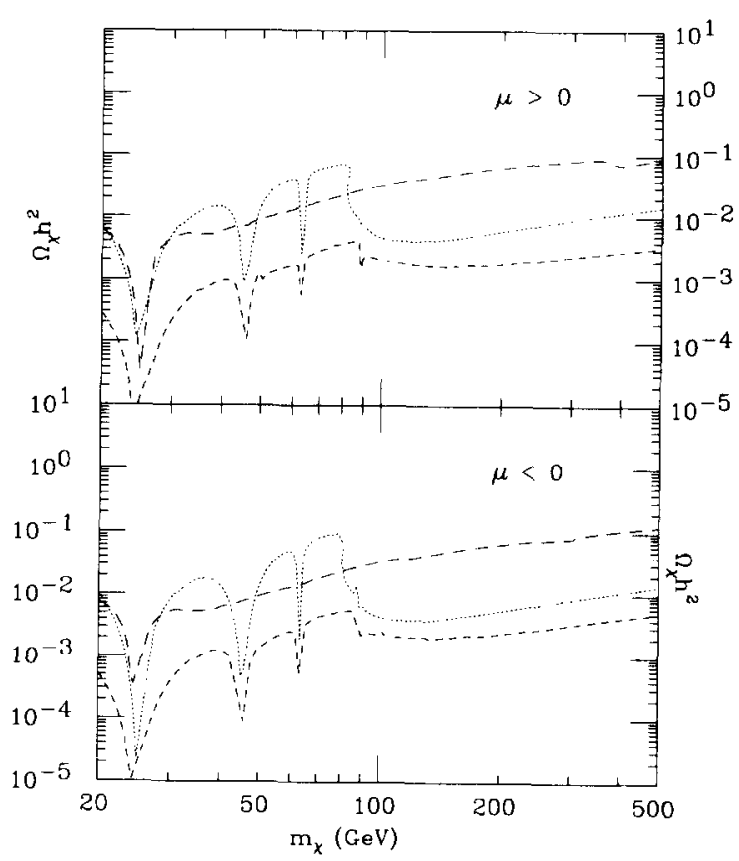

Fig. 2. Same as in Fig. 1, except that now compositions are: $P=0.01$ (dotted line), 0.5 (short-dashed line), 0.99 (long-dashed line).

evaluated according to the method presented in Ref. [8].

The annihilation rate $\Gamma_{A}$ is expressed in terms of the capture rate by the formula [9]

$$
\Gamma_{A}=\frac{C}{2} \tanh ^{2}\left(\frac{t}{\tau_{A}}\right)
$$

where $t$ is the age of the macroscopic body ( $t=$ $4.5 \mathrm{Gyr}$ for Sun and Earth), $\tau_{A}=\left(C C_{A}\right)^{-1 / 2}$, and $C_{A}$ is the annihilation rate per effective volume of the body, given by

$$
C_{A}=\frac{\langle\sigma v\rangle}{V_{0}}\left(\frac{m_{\chi}}{20 \mathrm{GeV}}\right)^{3 / 2} .
$$

$V_{0}$ is defined as $V_{0}=\left(3 m_{P l}^{2} T /(2 \rho \times 10 \mathrm{GeV})\right)^{3 / 2}$ where $T$ and $\rho$ are the central temperature and the central density of the celestial body. For the Earth $\left(T=6000 \mathrm{~K}, \rho=13 \mathrm{~g} \cdot \mathrm{cm}^{-3}\right) V_{0}=2.3 \times 10^{25} \mathrm{~cm}^{3}$, for the Sun $\left(T=1.4 \times 10^{7} \mathrm{~K}, \rho=150 \mathrm{~g} \cdot \mathrm{cm}^{-3}\right)$ $V_{0}=6.6 \times 10^{28} \mathrm{~cm}^{3} . \sigma$ is the neutralino-neutralino annihilation cross section and $v$ is the relative velocity. $\langle\sigma v\rangle$ is calculated with all the contributions at the tree 


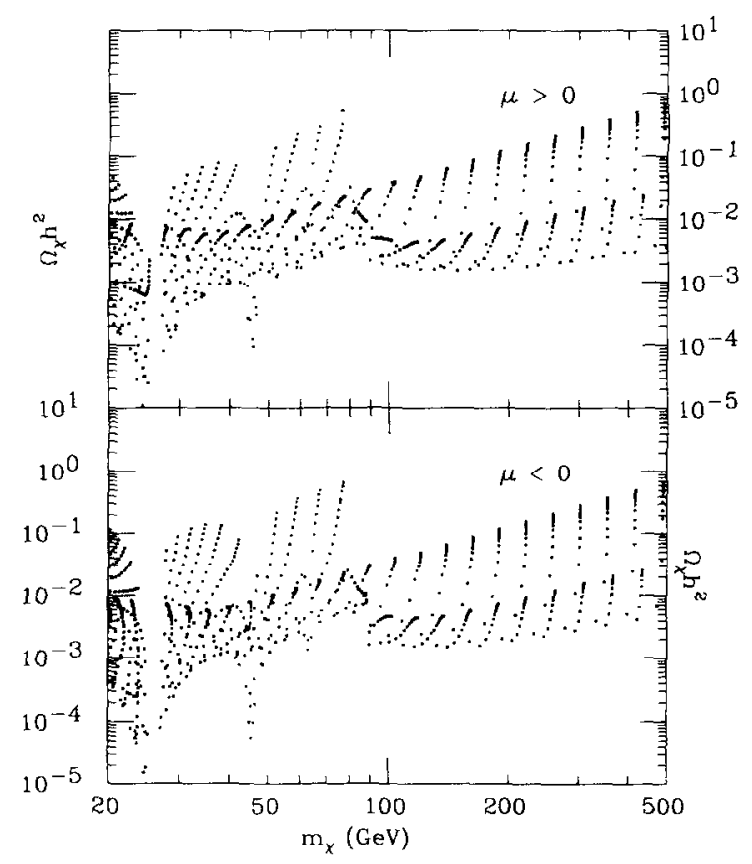

Fig. 3. Scatter plot for the neutralino relic abundance $\Omega_{\chi} h^{2}$ as a function of $m_{\chi}$. The two MSSM mass parameters $M_{2}, \mu$ are varied in the ranges: $20 \mathrm{GeV} \leq|\mu| \leq 3 \mathrm{TeV}, 20 \mathrm{GeV} \leq M_{2} \leq 6 \mathrm{TeV}$.

level as in Ref. [6], with the further inclusion here of the two gluon annihilation final state [10].

We recall that, according to Eq. (4), in a given macroscopic body the equilibrium between capture and annihilation (i.e. $\Gamma_{A} \sim C / 2$ ) is established only when $t \gtrsim \tau_{A}$. It is worth noticing that the neutralino density $\rho_{X}$, evaluated according to Eq. (2), enters not only in $C$ but also in $\tau_{A}$ (through $C$ ). Therefore the use of a correct value for $\rho_{X}$ (rescaled according to Eq. (2), when necessary) is important also in determining whether or not the equilibrium is already set in a macroscopic body.

In Fig. 4 we give the results of our calculations for $\tanh ^{2}\left(t / \tau_{A}\right)$ and for $\Gamma_{A}$ in the case of the Earth for three representative $\chi$-compositions: $P=$ $0.1,0.5,0.9$. For simplicity, in these figures, as well as in the following ones, only the results concerning positive values of the parameter $\mu$ are shown; similar results hold for negative values of $\mu$. The upper part of Fig. 4 shows that the equilibrium between capture and annihilation is not reached for $m_{X} \gtrsim m_{W}$; thus a substantial suppression occurs in $\Gamma_{A}$ at large values

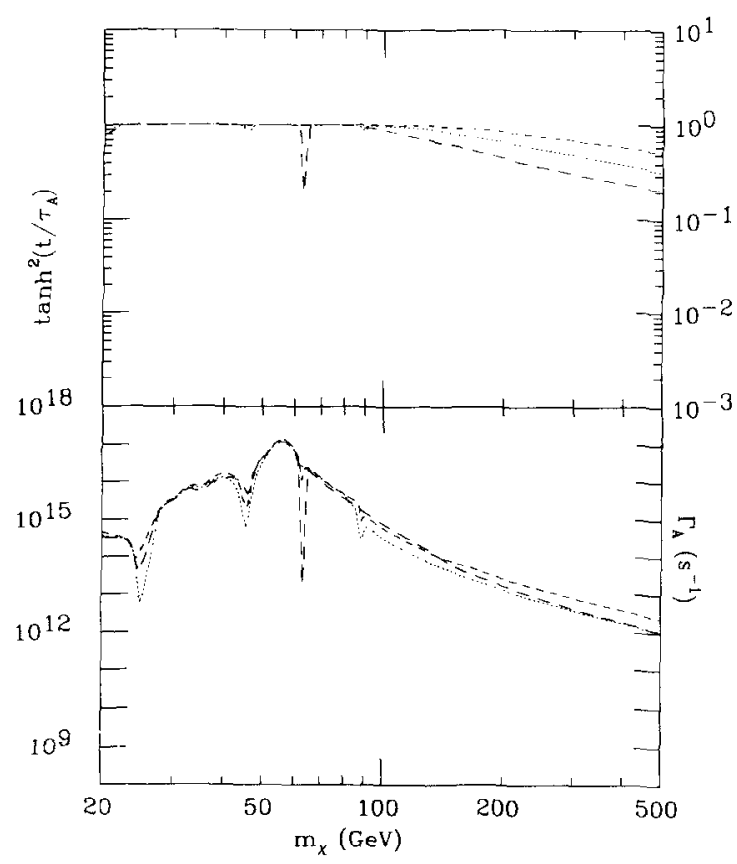

Fig. 4. $\tanh ^{2}\left(t / \tau_{A}\right)$ and $\Gamma_{A}$ for the Earth are given as functions of $m_{X}$, for the three representative neutralino compositions $P=0.1$ (dotted line), 0.5 (short-dashed line), 0.9 (long-dashed line).

of $m_{X}$ because of the factor $\tanh ^{2}\left(t / \tau_{A}\right)$. In Fig. 5 the two quantities $\tanh ^{2}\left(t / \tau_{A}\right)$ and $\Gamma_{A}$ are plotted for other representative values of neutralino compositions: $P=0.01,0.99$ (the value $P=0.5$ is shown again for comparison). In Figs. 4, 5 we see that, for $m_{\chi} \lesssim 70 \mathrm{GeV}, \Gamma_{A}$ shows the characteristic bumps due to fact that the capture is very efficient when the neutralino mass matches the nuclear mass of some important chemical component of the Earth $(\mathrm{O}, \mathrm{Si}$, $\mathrm{Mg}, \mathrm{Fe}$ ). Superimposed to these properties are the features due to the rescaling of $\rho_{\chi}$. In fact this rescaling implies here both a general suppression and the appearance of the peculiar dips reminiscent of the singularities in the annihilation cross section (discussed in Section 2). For comparison with Fig. 4 we show in Fig. 6 how the two quantities $\tanh ^{2}\left(t / \tau_{A}\right)$ and $\Gamma_{A}$ would appear, if the rescaling were not applied.

Whereas for the Earth the equilibrium condition depends sensitively on the values of the model parameters, in the case of the Sun equilibrium between capture and annihilation is reached for the whole range of $m_{\chi}$, due to the much more efficient capture rate im- 


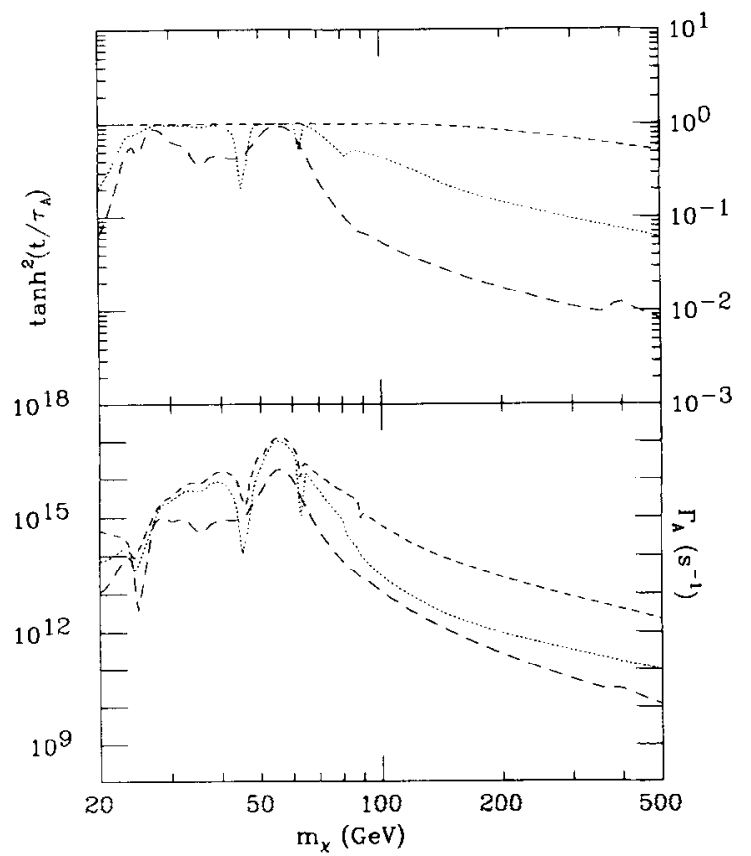

Fig. 5. Same as in Fig. 4, except that now compositions are: $P=0.01$ (dotted line), 0.5 (short-dashed line), 0.99 (long-dashed line).

plied by the stronger gravitational field [7]. $\Gamma_{A}$ for the Sun is shown in Fig. 7.

What we have defined above is the annihilation rate referred to a macroscopic body as a whole. This is certainly enough for the Sun which appears to us as a point source. On the contrary, in the case of the Earth, one has also to define an annihilation rate referred to a unit volume at point $r$ from the Earth center

$$
\Gamma_{A}(r)=\frac{1}{2}\langle\sigma v\rangle n^{2}(r)
$$

where $n(r)$ is the neutralino spatial density which may be written as [9]

$$
n(r)=n_{0} \mathrm{e}^{-\alpha m_{x} r^{2}}
$$

here $\alpha=2 \pi G \rho /(3 T)$ and $n_{0}$ is such that

$$
\Gamma_{A}=\frac{1}{2}\langle\sigma v\rangle \int d^{3} r n^{2}(r)
$$

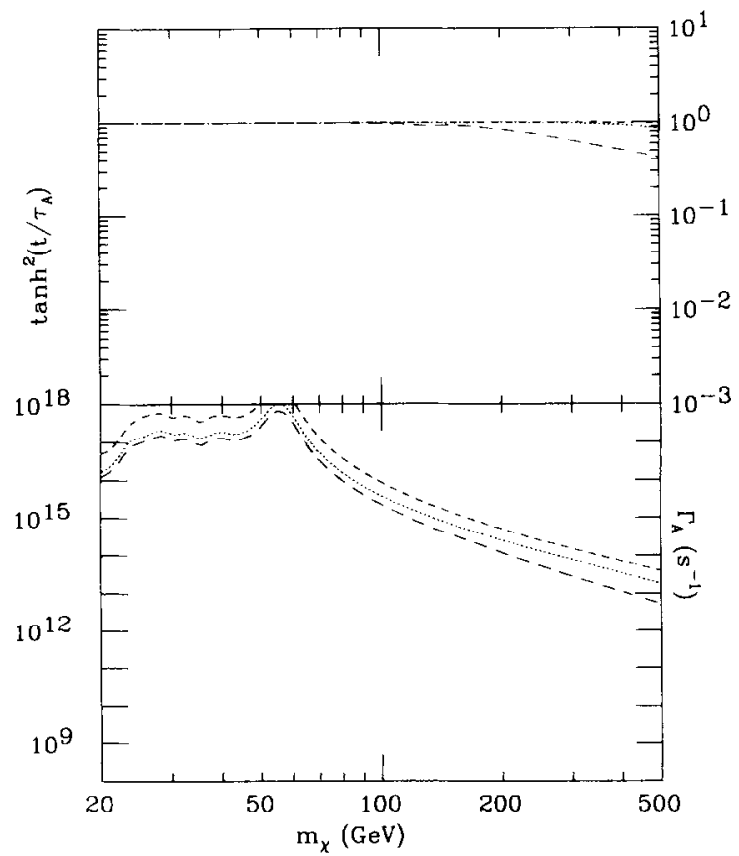

Fig. 6. Same as in Fig. 4, except that now the neutralino local density $\rho_{X}$ is not rescaled.

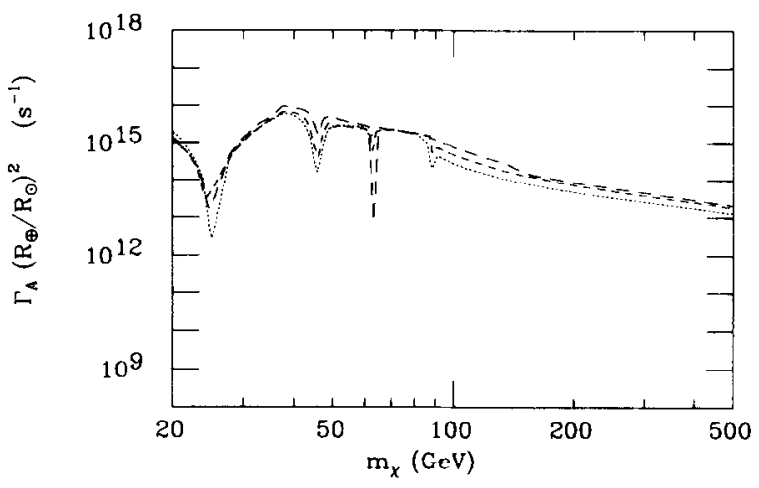

Fig. 7. Annihilation rate $\Gamma_{A}$ for the Sun as a functions of $m_{\chi}$, for the three representative neutralino compositions $P=0.1$ (dotted line), 0.5 (short-dashed line), 0.9 (long-dashed line). To easily compare the annihilation rate for the Earth to the one for the Sun, the plot shown here for the Sun refers to the effective annihilation rate, defined as $\Gamma_{A}$ times the ratio $\left(R_{\oplus} / R_{\odot}\right)^{2}=1.8 \times 10^{-9}$, where $R_{\oplus}$ is the radius of the Earth and $R_{\odot}$ is the Sun-Earth distance. 


\section{Neutrino fluxes}

The differential neutrino flux due to $\chi-\chi$ annihilation in a distant source (Sun) is given by

$$
\frac{d N_{\nu}}{d E_{\nu}}=\frac{\Gamma_{A}}{4 \pi d^{2}} \sum_{F, f} B_{\chi f}^{(F)} \frac{d N_{f \nu}}{d E_{\nu}}
$$

where $d$ is the distance from the source, $F$ denotes the $\chi-\chi$ annihilation final states, $B_{\chi f}^{(F)}$ denotes the branching ratios into heavy quarks, $\tau$ lepton and gluons in the channel $F ; d N_{f v} / d E_{\nu}$ is the differential distribution of the neutrinos generated by the hadronization of quarks and gluons and the subsequent hadronic semileptonic decays.

In the case of the Earth we define a differential neutrino flux generated at a distance $R$ by the $\chi-\chi$ annihilations which take place at a point $r$ from the center of the Earth

$$
\frac{d N_{\nu}}{d E_{\nu} d^{3} r}=\frac{\langle\sigma v\rangle n^{2}(r)}{8 \pi R^{2}} \sum_{F, f} B_{\chi f}^{(F)} \frac{d N_{f \nu}}{d E_{\nu}} .
$$

This is the neutrino spectrum which is used in our Monte Carlo code to evaluate the flux of up-going muons, as illustrated in Section 5.

However, it is also useful to write the neutrino differential flux at a point on the Earth surface, once the integration over the distribution $n(r)$ is performed, at fixed nadir angle $\theta[7]$

$$
\frac{d^{2} N_{\nu}}{d E_{\nu} d \cos \theta}=G(\theta) \frac{\Gamma_{A}}{4 \pi R_{\oplus}^{2}} \sum_{F, f} B_{\chi f}^{(F)} \frac{d N_{f \nu}}{d E_{\nu}} .
$$

$G(\theta)$ is given by

$$
G(\theta) \simeq 2(2 m \beta) \mathrm{e}^{-2 m \beta \sin ^{2} \theta}
$$

where $\beta=\alpha R_{\oplus}^{2}$, for $m_{X} \gtrsim 10 \mathrm{GeV}$ and $\theta \lesssim 60^{\circ}$. This flux, as all other fluxes discussed afterwards, refer to surfaces orthogonal to the incoming direction.

As far as the differential neutrino spectra are concerned, we briefly quote the relevant ingredients that we used in the calculation. A Jetset 7.2 Monte Carlo [11] code was used to compute the neutrino spectra due to $b$ and $c$ quarks, tau lepton and gluons. Following Ref. [12] we have neglected the contributions of the light quarks directly produced in the annihilation process or in the hadronization of heavy quarks

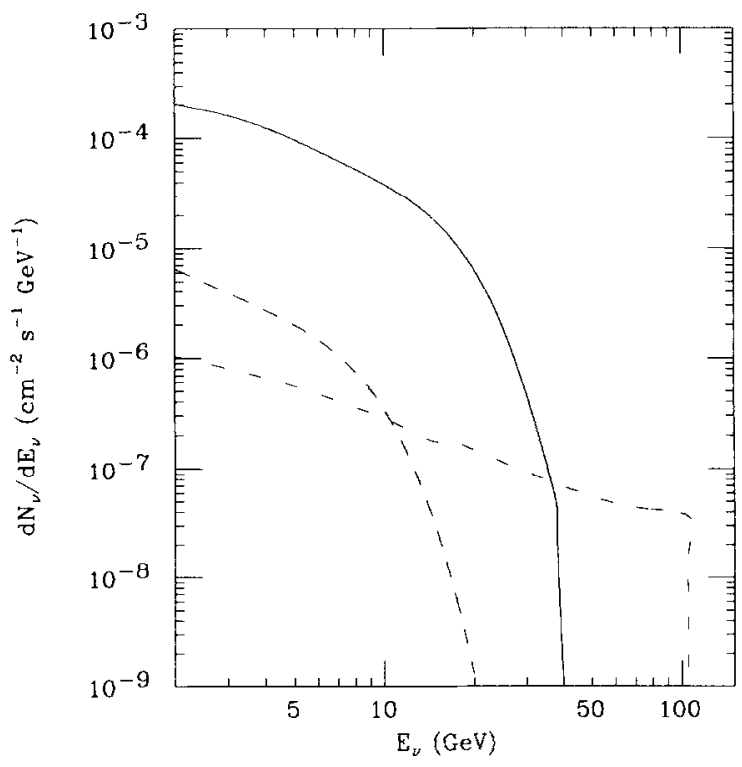

Fig. 8. Differential neutrino spectra $d N_{\nu} / d E_{\nu}$ for neutralino annihilation in the Earth, as a function of the neutrino energy $E_{\nu}$. These spectra are calculated for a neutralino composition $P=0.5$ and for three different values of neutralino mass: $m_{X}=20 \mathrm{GeV}$ (dashed line), $m_{X}=40 \mathrm{GeV}$ (solid line) and $m_{X}=120 \mathrm{GeV}$ (dash-dotted line).

and gluons, because these light particles stop inside the medium (Sun or Earth) before their decay. For the case of the Sun also the energy loss of the heavy hadrons in the solar medium was considered. The spectra due to heavier final states, i.e. Higgs bosons, gauge bosons and $t$ quark, were computed analytically by following the decay chain down to the production of a b quark, $c$ quark or a tau lepton; the result of the Monte Carlo was used to obtain the final neutrino output. Because of the high column density of the solar medium, the absorbtion and the energy loss of the produced neutrinos were also included.

As an example, we show in Fig. 8 the neutrino spectra for neutralino annihilation in the Earth, for $P=0.5$ and for three different values of the neutralino mass: $m_{\chi}=20,40$ and $120 \mathrm{GeV}$. The different shapes depend on which final states dominate in the annihilation cross section. For instance the spectrum at $m_{\chi}=120$ $\mathrm{GeV}$ shows the typical features due to the opening of the $W^{+}-W^{-}$final state in the $\chi-\chi$ annihilation. The relative magnitudes of the spectra at different values 
of $m_{\chi}$ are driven by the dependence of the annihilation rate $\Gamma_{A}$ on the model parameters.

\section{Signals of up-going muons}

Let us turn now to the calculation of the various distributions and rates for the up-going muons generated by the neutrino fluxes of Eqs. (9), (10) (signals) and by the neutrinos produced in the Earth atmosphere (background). The conversion of the muon neutrinos into muons and the subsequent muon propagation up to the detector have been treated using a Monte Carlo simulation.

The muon flux at the detector depends on the following quantities: the neutrino energy spectrum and spatial distribution, the differential $\nu_{\mu}-N$ charged current cross section for the muon production and the energy losses and the multiple scattering of the muon in the rock surrounding the detector.

As for the signals, the neutrino energies have been generated in the range $2 \mathrm{GeV} \leq E_{\nu} \leq m_{\chi}$ according to the distributions of Eq. (9) for the Sun and of Eq. (10) for the Earth. We emphasize that in the latter case the neutrino production point has been generated in a region around the Earth center with a gaussian distribution $n^{2}(r)$, where $n(r)$ is given in Eq. (7). The atmospheric neutrinos have been generated upwardly according to the differential distribution given by [13] for neutrinos with $E_{\nu} \leq 100 \mathrm{GeV}$ and by [14] for neutrinos with $E_{\nu}>100 \mathrm{GeV}$.

In the cross section for the charged current $\nu_{\mu}-N$ deep inelastic scattering we have employed the CTEQ parton distribution functions [15]. The energy losses and multiple scattering of the muon have been calculated by propagating the muon in standard rock by steps of $10^{4} \mathrm{~g} \mathrm{~cm}^{-2}$ when the muon energy was larger than $30 \mathrm{GeV}$, of $500 \mathrm{~g} \mathrm{~cm}^{-2}$ when it was between 1.2 and $30 \mathrm{GeV}$, and equal to $E_{\mu} /(2.4$. $\left.10^{-3} \mathrm{GeV}\right) \mathrm{g} \mathrm{cm}^{-2}$ for the final step when $E_{\mu}<$ $1.2 \mathrm{GeV}$. The residual energy and the muon direction were kept in memory for each step from the muon production to the muon stopping. The detector entry point has been randomly generated with a uniform probability along the whole muon track. The muon energy and direction at the entry point has been calculated by interpolating between the initial and final values for the step where the entry point occurred. A random an-
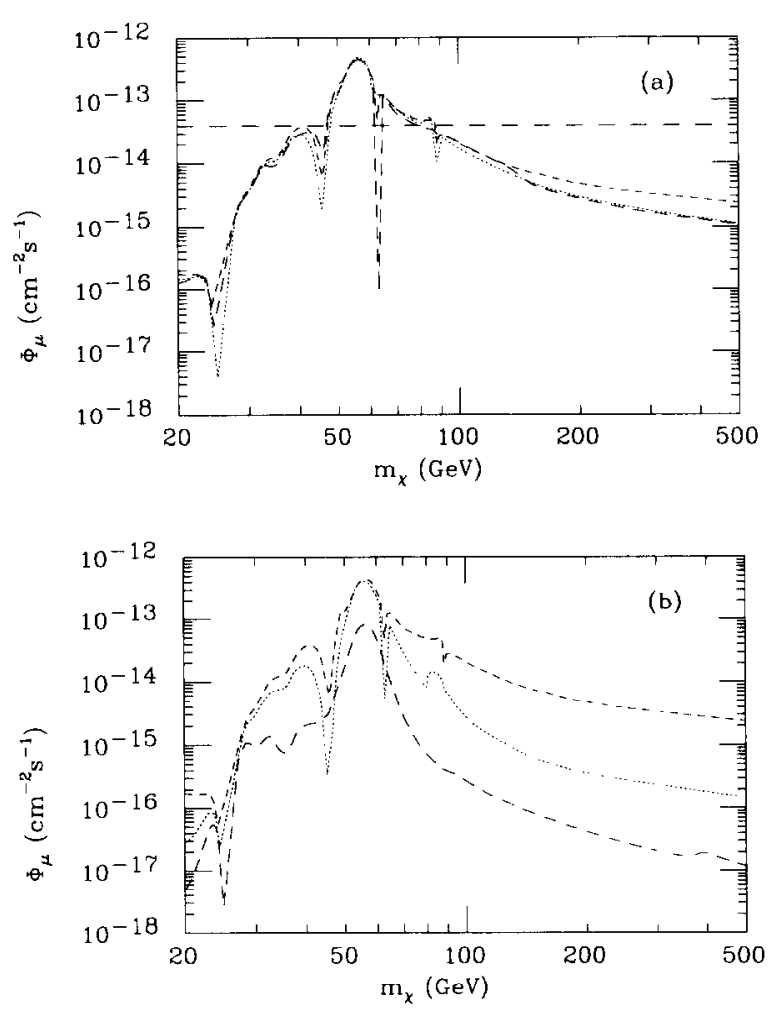

Fig. 9. Flux $\Phi_{\mu}$ of the up-going muons as functions of $m_{\chi}$ for $\chi-\chi$ annihilation in the Earth. The threshold for the muon energy is $E_{\mu}^{\text {th }}=2 \mathrm{GeV}$. In figure (a) the three representative neutralino compositions are $P=0.1$ (dotted line), 0.5 (short-dashed line), 0.9 (long-dashed line). In figure (b) $P=0.01$ (dotted line), 0.5 (short-dashed line), 0.99 (long-dashed line). The horizontal line in figure (a) represents the Kamiokande upper bound $4.0 \cdot 10^{-14} \mathrm{~cm}^{-2} \mathrm{~s}^{-1}$ (90\% C.L.) [2].

gle has been included to take into account a detector angular resolution of $2^{\circ}$.

\section{Results and conclusions}

We show in Figs. 9-11 a sample of our results for the signals expected from the Earth at the usual representative point $\tan \beta=8$ (for the fluxes and the angular distributions shown in these figures and in the following ones the threshold for the muon energy is set at the value $E_{\mu}^{\text {th }}=2 \mathrm{GeV}$ ). Fig. 9 displays the integral muon flux versus $m_{\chi}$ for various neutralino compositions. This has been obtained with a no-straggling approximation [16], i.e. the muons are produced and propagated in the forward direction (when this ap- 


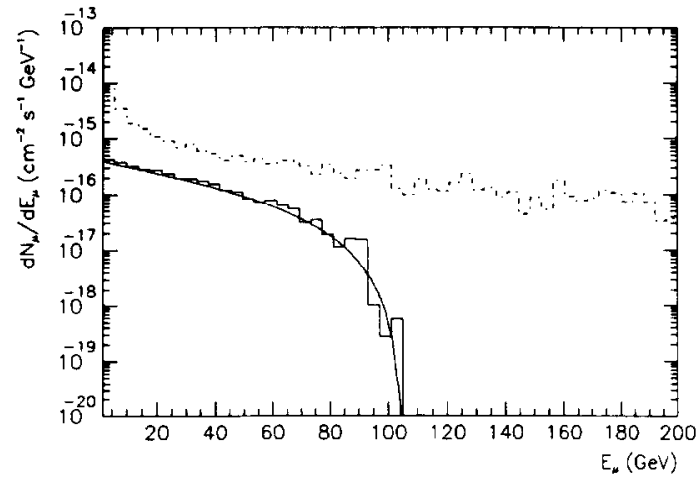

Fig. 10. Differential muon spectra $d N_{\mu} / d E_{\mu}$ as a function of the muon energy $E_{\mu}$. The solid histogram is the muon spectrum obtained with our MC simulation for $P=0.5$ and $m_{X}=120$ $\mathrm{GeV}$. The solid line represents the muon spectrum calculated in the no-straggling approximation. The dash-dotted histogram is the muon energy distribution for the background of atmospheric neutrinos. The spectra refer to muons entering the detector within a cone of half-aperture of $30^{\circ}$ centered at the nadir.

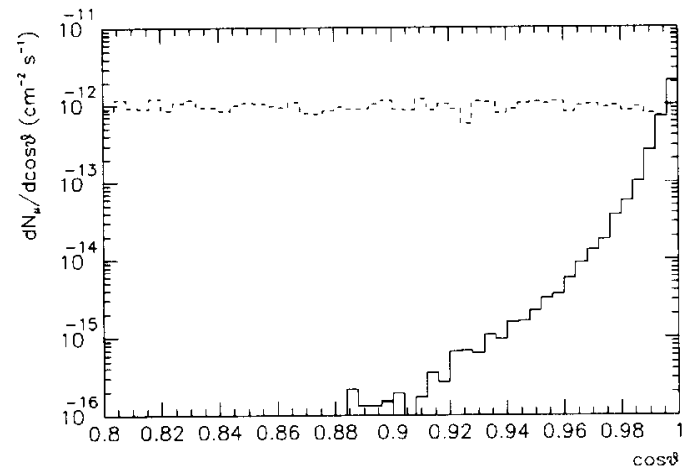

Fig. 11. Muon angular distribution $d N_{\mu} / d \cos \theta$ as a function of $\cos \theta$, where $\theta$ is the nadir angle. The solid histogram is the angular distribution of the signal obtained with our MC simulation for $P=0.5$ and $m_{\chi}=120 \mathrm{GeV}$. The dashed histogram is the muon angular distribution for the background of atmospheric neutrinos.

proximation is applied, then Eq. (11) for the neutrino distribution is useful ). The general structure of the flux clearly reflects the features of the annihilation rate in Earth, previously discussed. The horizontal line represents the Kamiokande upper bound for signals coming from the Earth [2]: $4.0 \cdot 10^{-14} \mathrm{~cm}^{-2} \mathrm{~s}^{-1}$ (90\% C.L.); this value refers to a half-aperture of $\theta_{t}=30^{\circ}$ for a cone centered at the nadir. By comparing this upper limit with our fluxes we see that the regions explored by Kamiokande (at our representative point: $\tan \beta=$
$8, m_{h}$ and $m_{\tilde{f}}$ chosen as stated in Section 1 ) concern the neutralino mass range $50 \mathrm{GeV} \lesssim m_{\chi} \lesssim 80 \mathrm{GeV}$, for $0.1 \lesssim P \lesssim 0.9$; the mass range is narrower for purer neutralino compositions.

By way of example we show in Figs. 10, 11 the differential spectra and the angular distributions for the case $P=0.5$ and $m_{\chi}=120 \mathrm{GeV}$. In Fig. 10 the spectra obtained with the MC code for the expected signal and for the background are displayed by the two histograms. The solid line represents the spectrum for the signal obtained with the no-straggling approximation; it is clear from the plot that this approximation is quite good for the spectrum, and then also for the integrated flux. The spectra shown in this figure refer to the muons entering the detector within a cone of half-aperture $\theta_{t}=30^{\circ}$ centered at the nadir. In Fig. 11 the angular distribution due to the signal, markedly peaked around the nadir direction, is compared with the almost flat background distribution.

Now a very relevant question is what is the minimal exposure At necessary to provide a good S/B discrimination. To put it into a more quantitative way, we define an $(A t)_{\min }$ as the minimal exposure necessary to see a $4 \sigma$ effect (with a signal of at least 4 events) [17]. (At $)_{\min }$ is displayed in Fig. 12a (the angle $\theta_{t}$ of half-aperture from the nadir is again set at the value $\theta_{t}=30^{\circ}$ here). However, it is important to remark that, due to the different features of the angular distributions for the signal and for the background (illustrated for instance in Fig. 11), (At $)_{\min }$ may be optimized by appropriately choosing the angular window, i.e. $\theta_{t}$. We call $(A t)_{\mathrm{opt}}$ the least value of $(A t)_{\mathrm{min}}$ when $\theta_{t}$ is varied, and we denote by $\theta_{\mathrm{opt}}$ the corresponding value of $\theta_{t}$. This optimization procedure has to be applied to $(A t)_{\min }$ for each value of the integral flux $\Phi_{\mu}$. For the case illustrated in Fig. 11 we find $\theta_{\mathrm{opt}} \simeq 5^{\circ},(A t)_{\mathrm{opt}} \simeq 3020 \mathrm{~m}^{2}$ yr. Fig. $12 \mathrm{~b}$ displays the dependence of $(A t)_{\mathrm{opt}}$ on $m_{X}$ and $P$ for our usual representative case. To obtain the result of Fig. 12b the optimization, based on the angular distribution, has been performed by using the results of the MC simulations at 4 values of the neutralino mass: $m_{X}=20$, $40,60,120 \mathrm{GeV}$, by interpolating between these values and by conservatively using for $m_{X}>120 \mathrm{GeV}$ the same angular window as for $m_{\chi}=120 \mathrm{GeV}$. For the integral flux we employed the results (shown in Fig. 9a) obtained with the no-straggling approximation. By comparing Figs. 12a and $12 \mathrm{~b}$ one sees that the 

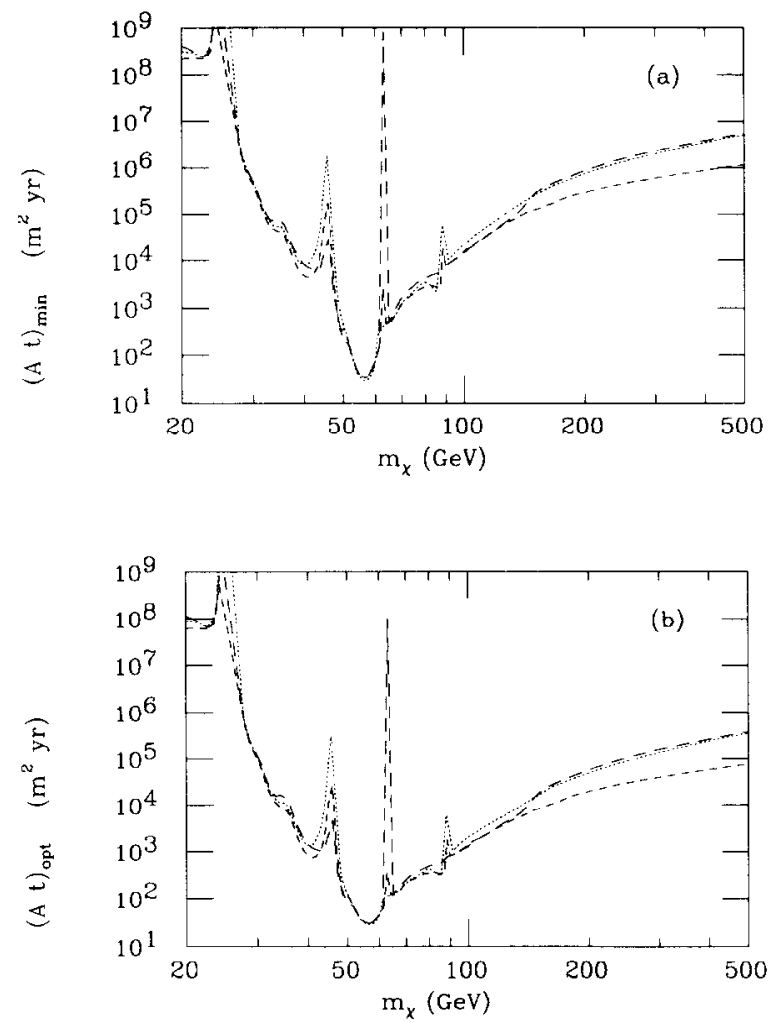

Fig. 12. Exposure necessary to have a $4 \sigma$ effect with a signal of at least 4 events, as a function of neutralino mass $m_{X}$. The figure refers to a signal coming from the Earth. The three representative neutralino compositions are $P=0.1$ (dotted line), 0.5 (short-dashed line), 0.9 (long-dashed line). In figure (a) $A t_{\min }$ is displayed keeping the half-aperture angle of the detector fixed at $30^{\circ}$ from the center of the Earth. Figure (b) displays the exposure $A t_{\text {opt }}$ obtained by employing the optimization procedure.

optimization improves significantly the experimental capabilities especially at large $m_{X}$ values; in fact for these values the extension of the annihilation region is very much concentrated around the Earth center. Fig. $12 \mathrm{~b}$ shows realistically by which amount the explorable ranges of the two neutralino parameters $m_{X}$ and $P$ increase as the exposure $A t$ is increased. We remind that the largest value for $A t$ obtained up to now is the one of Kamioka: $A t=770 \mathrm{~m}^{2}$ yr [2]. A sizeable improvement in $A t$ will be provided by MACRO [18].

Let us turn now to the signals from the Sun. In Fig. 13 the relevant flux of up-going muons is plotted versus $m_{\chi}$ in the case of the representative point under discussion; $\Phi_{\mu}$ is the flux through a surface orthogonal

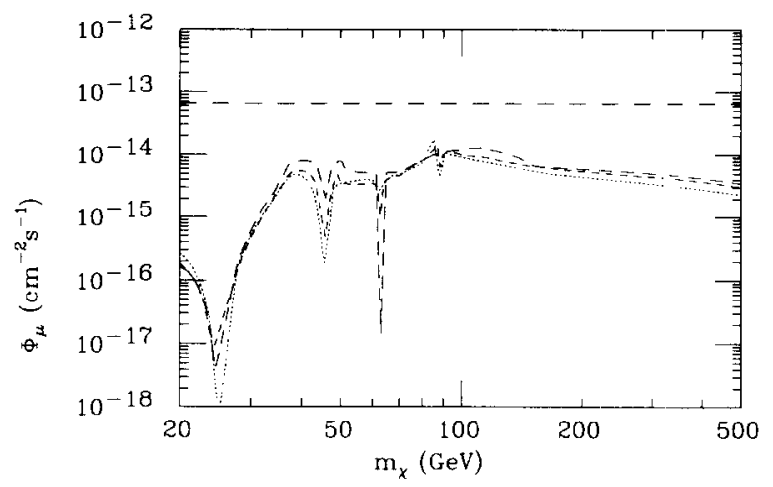

Fig. 13. Flux $\Phi_{\mu}$ of the up-going muons as functions of $m_{\chi}$ for $\chi-\chi$ annihilation in the Sun. The flux is orthogonal to the Sun direction and the threshold for the muon energy is $E_{\mu}^{\mathrm{th}}=2 \mathrm{GeV}$. The three representative neutralino compositions are $P=0.1$ (dotted line), 0.5 (short-dashed line), 0.9 (long-dashed line). The horizontal line represents the Kamiokande upper bound $6.6 \cdot 10^{-14} \mathrm{~cm}^{-2} \mathrm{~s}^{-1}$ (90\% C.L.) [2].

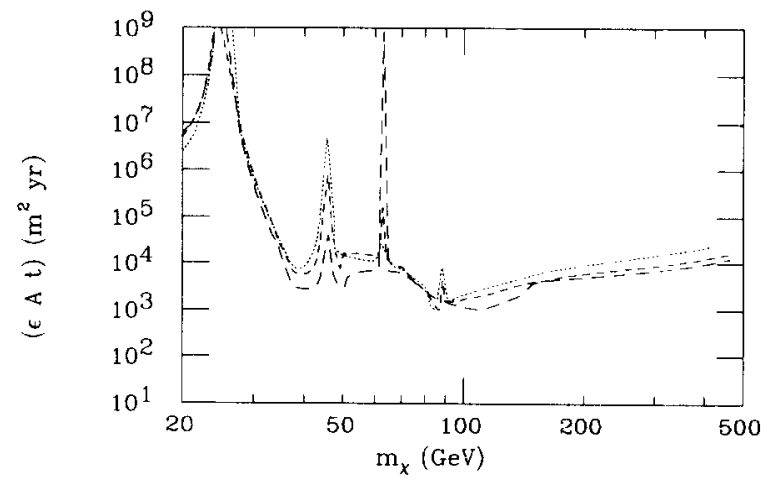

Fig. 14. Effective exposure ( $\epsilon A t)$ necessary to have a $4 \sigma$ effect with a signal of at least of 4 events, as a function of neutralino mass $m_{\chi}$. The figure refers to a signal coming from the Sun. The three representative neutralino compositions are $P=0.1$ (dotted line), 0.5 (short-dashed line), 0.9 (long-dashed line). The half-aperture of the detector is fixed at $5^{\circ}$ from the Sun direction.

to the Sun direction. The horizontal line denotes the present upper bound of $6.6 \cdot 10^{-14} \mathrm{~cm}^{-2} \mathrm{~s}^{-1}(90 \%$ C.L.) (an half-aperture $\theta=25^{\circ}$ was taken here for a cone centered at the Sun direction) [2]. It is clear that this limit does not introduce any constraint here. In Fig. 14 the corresponding $(\epsilon A t)-m_{X}$ plot is displayed (a cone of $5^{\circ}$ half-aperture around the Sun direction has been used for the evaluation of the background, $\epsilon$ is the on-source duty factor). In this plot we see that, in order to start some exploration about relic neutralinos 
using the flux from the Sun, one needs at least $(\epsilon A t) \simeq$ $10^{4} \mathrm{~m}^{2} \mathrm{yr}$ (this could provide information in the range $\left.70 \mathrm{GeV} \lesssim m_{\chi} \lesssim 150 \mathrm{GeV}\right)$. However, it is remarkable that by slightly increasing ( $\epsilon A t)$ above this level, the explorable range in terms of $P$ and $m_{X}$ expands quite rapidly towards the high values of the neutralino mass.

An interesting point concerns the relative importance of the up-going muon fluxes from the Earth and from the Sun. As we see from Figs. 9, 13, for light neutralinos the signal from the Sun is much smaller than the signal from the Earth; as $m_{X}$ increases the signal from the Sun may overcome the other, since the Sun gravitational field is much more efficient in capturing the neutralinos. More relevant for a comparative discussion about the signals from the two sources are the two diagrams of the exposure versus $m_{\chi}$ displayed in Figs. 12b, 14. A very appealing situation is the one where the signals from both sources could be detected. For instance, assuming $\epsilon \simeq 0.5$, an exposure $A t \simeq 10^{5} \mathrm{~m}^{2}$ yr would be sensitive to both signals in the range $35 \mathrm{GeV} \lesssim m_{\chi} \lesssim 200 \mathrm{GeV}$.

A word of warning is in order here to remind that the present evaluations depend sensitively on a number of free parameters. As far as $\tan \beta$ is concerned, we may notice that the dependence of the signals on this parameter is rather involved, but, as a general trend, the expected signals increase with the value of $\tan \beta$. For instance, for the Sun, moving from the value $\tan \beta=8$ to the value $\tan \beta=20$ the flux is enhanced by about a factor 5 for $m_{\chi} \gtrsim 200 \mathrm{GeV}$; but, if the value of $\tan \beta$ is reduced from $\tan \beta=8$ to $\tan \beta=2$, the flux is suppressed (for $m_{\chi} \gtrsim 50 \mathrm{GeV}$ ) roughly by an order of magnitude. The quantities calculated in the present paper are also rather sensitive on the values employed for the Higgs and the sfermion masses. For instance, our signals for the Earth are roughly proportional to $m_{h}^{-4}$. In the present paper we have used for the Higgs and the sfermion masses values close to their present lower bounds. This choice enables one to establish, by use of the figures previously reported, what is the minimum exposure necessary for a detector to be sensitive to the Earth and/or Sun signals and how the exploration into the DM neutralino properties may be progressively improved by expanding the size of the experimental apparatus.

To summarize we may conclude that the exploration in the realm of the relic neutralinos by neutrino telescopes, after the first insights provided by Kamiokande
[2] about the Earth signals, will certainly be significantly developed by the underwater (under-ice) detectors under construction [3]. In order to have good perspectives for a systematic investigation of this field one should finally aim at an apparatus of very large area of order of $10^{6} \mathrm{~m}^{2}$.

\section{Acknowledgments}

We are grateful to Professor V.S. Berezinsky for very interesting discussions.

\section{References}

[1] G.F. Giudice and E. Roulet, Nucl. Phys. B 316 (1989) 429; G.B. Gelmini, P. Gondolo and E. Roulet, Nucl. Phys. B 351 (1991) 623;

M. Kamionkowski, Phys. Rev. D 44 ( 1991) 3021:

A. Bottino, V. de Alfaro, N. Fornengo, G. Mignola and M. Pignone, Phys. Lett. B 265 (1991) 57;

F. Halzen, M. Kamionkowski and T. Steltzer, Phys. Rev. D 45 ( 1992) 4439;

V.S. Berezinsky, Nucl. Phys. (Proc. Suppl.) B 31 (1993)

413 (Proc. Neutrino 92, Ed. A. Morales)

M. Mori et al., Phys. Rev. D 48 (1993) 5505;

M. Drees, G. Jungman, M. Kamionkowski and M.M. Nojiri, Phys. Rev. D 49 (1994) 636;

R. Gandhi, J.L. Lopez, D.V. Nanopoulos, K. Yuan and A. Zichichi, Phys. Rev. D 49 (1994) 3691

[2] M. Mori et al. (Kamiokande Collaboration), Phys. Lett. B 289 (1992) 463.

|3] J.G. Learned, Nucl. Phys. (Proc. Suppl.) B 31 (1993) 456 (Proc. Neutrino 92, Ed. A. Morales);

F. Halzen, Proc. Fourth International Workshop on Neutrino Telescopes (1992), Ed. M. Baldo Ceolin;

G.V. Domogatsky, Nucl. Phys. (Proc. Suppl.) B 35 (1994) 290 (Proc. TAUP 93, Ed.s C.Arpesella, E.Bellotti, A.Bottino);

L.K. Resvanis, loc. cit. 294.

[4] H.P. Nilles, Phys. Rep. 110 ( 1984) 1; H.E. Haber and G.L. Kane, Phys, Rep. 117 (1985) 75; R. Barbieri, Rivista Nuovo Cimento 11 (1988) 1.

[5] T.K. Gaisser, G. Steigman and S. Tilav, Phys. Rev. D 34( 1986$) 2206$.

[6] A. Bottino, V.de Alfaro, N. Fornengo, G. Mignola and M. Pignone, Astroparticle Physics 2 (1994) 67.

[7] A. Gould, Ap. J. 321 (1987) 571; Ap. J. 328 (1988) 919; Ap. J. 368 (1991) 610.

18] A. Bottino, V. de Alfaro, N. Fornengo, A. Morales, J. Puimedón and S. Scopel, Mod. Phys. Lett. A 7 (1992) 733. Notice that in the present paper for the Higgs boson-nucleon coupling we use the evaluation of J. Gasser, H. Leutwyler and M. E. Sainio, Phys. Lett. B 253 (1991) 252. 
[9] K. Griest and D. Seckel, Nucl. Phys. B 283 (1987) 681.

[10] M. Drees, G. Jungman, M. Kamionkowski and M.M. Nojiri, Phys. Rev. D 49 (1994) 636.

[11] T. Sjöstrand, Comp. Phys. Comm. 39 (1986) 347; Comp. Phys. Comm. 43 (1987) 367; CERN-TH 6488/92.

[12] S.Ritz and D.Seckel, Nucl. Phys. B 304 (1988) 877.

[13] G. Barr, T.K. Gaisser and T. Stanev, Phys. Rev. D 39 (1989) 3532 and private communication.

[14] L.V. Volkova, Sov. J. Nucl. Phys. 31 (1980) 784.

[15] J. Botts, J.G Morfin, J.F. Owens, J. Qiu, W. Tung and H. Weerts, Phys. Lett. B 304 (1993) 159.
[16] T.K.Gaisser: Cosmic Rays and Particle Physics (Cambridge University Press, 1990).

[17] A more refined statistical treatment of the $S$ /B discrimination will be presented by the present authors in a forthcoming paper.

[18] D.G.Michael (MACRO Collaboration), Nucl. Phys. (Proc. Suppl.) B 35 (1994) 235 (Proc. TAUP 93, Eds. C.Arpesella, E.Bellotti, A.Bottino). 